\title{
Bibliotecas y visualización de la información: análisis del mapa de la lectura pública en España basado en la herramienta Secaba-Rank a través de su representación gráfica
}

\author{
Pedro Lázaro Rodríguez ${ }^{1}$ \\ ${ }^{1}$ Código ORCID: http://orcid.org/0000-0002-8756-0507. Universidad de Granada. Departamento \\ de Información y Comunicación. Campus Universitario de Cartuja. Edificio Máximo. 18071 \\ Granada, España. Correo electrónico: pedrolr@ugr.es.
}

Tipo de contribución: comunicación

Resumen: Se propone un análisis del mapa de la lectura pública en España (bibliotecas públicas) basado en la metodología Secaba-Rank sobre evaluación de bibliotecas a nivel de comunidades autónomas. Dicha metodología mide la eficiencia de bibliotecas gracias al indicador de segundo nivel llamado Potencia del Sistema. Este indicador pone en relación las entradas de los sistemas biblioteca (presupuesto) y las salidas (préstamos, visitas, etc.). Se ofrecen 2 representaciones gráficas para analizar el mapa: la primera, se basa en un diagrama de dispersión que, gracias a la línea de tendencia, permite distinguir qué sistemas obtienen unas salidas mejor de lo esperado y cuáles peor; la segunda, se basa en un gráfico en movimiento a modo de circunferencia que permite relacionar la eficiencia de los sistemas con la fórmula matemática de la circunferencia y el espacio que recorren cada uno de los mismos. A su vez, esta segunda representación se relaciona con una de las 5 leyes para las bibliotecas formuladas por Ranganathan (la biblioteca es un organismo en crecimiento). En las conclusiones se lleva a cabo una reflexión acerca de todos los temas tratados. El valor y originalidad del trabajo se entienden por cuanto supone una nueva forma de visualización de la información en relación a bibliotecas y su evaluación para el mejor entendimiento y apreciación de la realidad para su mejora.

Palabras clave: Lectura Pública: Bibliotecas Públicas; Visualización de la Información; Mapa de la Lectura; Potencia del Sistema; Secaba-Rank.

Abstract: The current work includes an analysis of the map of public reading in Spain (public libraries) at the level of Autonomous Communities based on the Secaba-Rank methodology for the evaluation of libraries. This methodology measures the efficiency of libraries thanks to the second level indicator named as System Potency. This indicator takes into account the relation between library system inputs (budget) and outputs (loans, visits, etc.). Two graphical representations are shown to analyze the map: the first is based on a scatter plot chart which allows to distinguish which systems have better than expected outputs and which have worse; the second representation is based on a moving chart as a circumference which allows to relate the efficiency of the systems with the mathematical formula of the circumference. Also, a reflection is carried out on one of the laws for libraries formulated by Ranganathan (the library is a growing organism). The value and originality of the current work is that it presents a new form for the information visualization on libraries and the evaluation to understand their current situation in a better way.

Keywords: Public Reading: Public Libraries; Information Visualization; Reading Maps; System Potency; Secaba-Rank. 


\section{Introducción}

En 2016 se desarrolló e implementó Secaba-Rank ${ }^{1}$, herramienta en línea y abierta cuyo objetivo es la evaluación de las redes de lectura pública (bibliotecas públicas) y las bibliotecas universitarias de España. La metodología Secaba-Rank se basa en el desarrollo de indicadores de segundo nivel para medir la eficiencia desde la perspectiva de la teoría general de sistemas (enfoque sistémico). La herramienta supuso la elaboración de un ranking de eficiencia con actualización anual permitiendo la identificación de los modelos de buenas y el benchmarking en aras a la mejora especialmente de los sistemas o bibliotecas que más lo necesitan. La herramienta fue presentada y difundida a la comunidad científica a inicios del año 2018 (Lázaro-Rodríguez, López-Gijón, Alonso, Martínez-Sánchez y Herrera-Viedma, 2018) y comparada en 2019 con otras técnicas para medir la eficiencia como lo son la técnica DEA (Análisis Envolvente de Datos) o el método Finlandia (Lázaro-Rodríguez, López-Gijón y Herrera-Viedma, 2019).

Una sección de la herramienta Secaba-Rank que adquiere especial relevancia por la representación gráfica de la información que contiene es la nombrada como Estimación y Predicción ${ }^{2}$. En dicha sección se ofrece un mapa de la lectura basada en la representación gráfica y visualización de la relación entre el presupuesto de las bibliotecas (sistemas) y sus salidas (circulación o préstamos). Gracias al diagrama de dispersión y la línea de tendencia, se distingue claramente qué sistemas son los que obtienen unas salidas mejor de lo esperado y cuáles quedan por debajo de lo esperado. Además, el mapa está diseñado según un sentido dinámico permitiendo la interacción con el mismo gracias a la tecnología de HighCharts $\mathrm{Cloud}^{3}$ con la que fue elaborado.

Otra representación gráfica que puede plantearse del mismo mapa es la basada en el sentido de la circunferencia o gráfico de radar mostrando a los sistemas en movimiento. Esta representación gráfica no está incluida en la herramienta y por ello puede considerarse como un aporte de originalidad del presente trabajo. Como se verá, esta forma innovadora de visualizar información relacionada a bibliotecas permite dar carácter matemático a los resultados gracias a la fórmula matemática del radio de la circunferencia y el espacio recorrido por cada sistema en una misma unidad de tiempo. Además, dado que los sistemas no eficientes girarían en sentido contrario al de las agujas del reloj simulando el sentido negativo, esta representación gráfica permite traer a colación una de las 5 leyes para las bibliotecas establecidas por el matemático indio Ranganathan, a saber: que las bibliotecas son un organismo en crecimiento, esto es, en expansión, y no en implosión (Ranganathan, 1931). Así, se puede plantear la cuestión de si los sistemas que están en implosión y que serían los que se mueven en sentido negativo, pueden ser considerados de hecho bibliotecas.

En este trabajo se analiza el mapa de la lectura pública ofrecido por la herramienta Secaba-Rank en base a dos representaciones gráficas: la incluida en la sección antes mencionada de la Estimación y Predicción representa; y su representación gráfica en movimiento en base a la idea de circunferencia y de sentido matemático. Aunque la herramienta Secaba-Rank incluye Secaba-Rank Universitarias y Secaba-Rank Públicas, en este trabajo se toma en consideración el mapa de la lectura pública en España, esto es, el incluido en Secaba-Rank Públicas. A su vez, aunque SecabaRank incluye un doble nivel de análisis a nivel de comunidades autónomas y provincias, en este trabajo se considera el mapa de la lectura pública a nivel de comunidades autónomas.

1. Acceso desde: $\mathrm{http} / / /$ secaba.ugr.es/rank/

2. Acceso desde: http://secaba.ugr.es/rank/publicas/index.php/estimacion-y-prediccion/

3. Acceso desde: https://cloud.highcharts.com/ 
Para ello, a continuación se enumeran 3 objetivos y preguntas de investigación. La siguiente sección incluye un repaso al concepto de mapa de la lectura de bibliotecas en España a modo de breve revisión de la literatura mostrando algunos ejemplos existentes y definiendo lo que Secaba-Rank entiende por mapa de la lectura. La sección 4 está dedicada a la especificación de los materiales y metodología que sigue este trabajo. A continuación, se muestran los resultados o mapas de la lectura en que se basa el trabajo, para finalizar con una sección para la discusión y las conclusiones.

\section{Objetivos y preguntas de investigación}

Los objetivos de la comunicación pueden entenderse como la respuesta a las siguientes preguntas de investigación:

- ¿Cuáles son los resultados de un análisis del mapa de la lectura pública en España a nivel de comunidades autónomas ofrecido en la herramienta Secaba-Rank?

- ¿Qué redes, sistemas o bibliotecas quedan por encima de lo esperado y cuáles por debajo en la relación entre entradas (presupuesto) y salidas (préstamos, visitas, etc.)?

- ¿Cuáles son las ventajas de las nuevas formas de visualización de la información propuestas en el campo de las bibliotecas en particular y de la documentación en general?

\section{Breve revisión de la literatura}

El concepto de mapa de la lectura o de bibliotecas en España tiene una naturaleza estrechamente relacionada con su evaluación para alcanzar conclusiones en torno a su situación actual y la posible mejora de los servicios que ofrecen. Por ejemplo, el mapa de la lectura pública valenciana se considera como un instrumento de trabajo para la planificación acometiendo el estudio de las necesidades bibliotecarias y su planificación (Galán-Serrano, Lloret, Peset-Mancebo, Torres Faus, 2002).

En un sentido similar, se disponen de otros mapas de la lectura pública como el de Cataluña (Portús-i-Vinyeta, 1999) o el del concejo de Mieres (González-Rodríguez et al., 2005). Por ejemplo, el segundo se marcaba los objetivos de ser un mapa de recursos recogiendo la distribución del servicio bibliotecario de Mieres, definiendo estándares del servicio de biblioteca pública propuesto para el concejo atendiendo a los equipamientos futuros del servicio bibliotecario para la población, y proponiendo un instrumento de planificación para la gestión y promoción del desarrollo de los servicios.

De entre los mapas disponibles en España, el que más cabe resaltar es el propuesta por Javier López Gijón para la comunidad autónoma de Andalucía (López-Gijón, 2003). En dicho trabajo se desarrolló lo que pueden considerarse como los cimientos de la metodología Secaba-Rank para medir la eficiencia de bibliotecas e incluye gráficos de correlaciones entre el presupuesto de las bibliotecas y los préstamos.

Esa misma idea es en la que se basa el mapa de la lectura pública por comunidades autónomas en que se basa el actual trabajo. El mapa puede entenderse, planteando un símil, como el mapa de una ciudad que se utiliza para poder movernos por la misma. Así, el mapa de la lectura pública a nivel de comunidades autónomas planteado desde la eficiencia y propuesto en Secaba-Rank ha de entenderse como un instrumento para saber moverse por la realidad de las bibliotecas públicas, y 
especialmente en lo que a gestión, mejora y toma de decisiones se refiere. Además, el mapa de la lectura pública ofrecido por Secaba-Rank no es solo un medio o parte de la evaluación de bibliotecas, sino un original aporte a la mejora de las mismas basado en nuevas formas de visualizar la información para su mejor apreciación y entendimiento (Chen, 2006; Yau, 2013).

\section{Materiales y metodología}

Los materiales utilizados son los resultados de la herramienta Secaba-Rank para las bibliotecas públicas (Secaba-Rank Públicas ${ }^{4}$ ), en concreto los resultados del mapa de la lectura pública ofrecido en la sección de Estimación y Predicción ${ }^{5}$ para los dos últimos años (2015 y 2016) que incluye y a nivel de comunidades autónomas. Los principales resultados de Secaba-Rank que se utilizaron para elaborar las representaciones gráficas y que se utilizarán para comentarlas se incluyen en la Tabla 1.

Tabla 1. Resultados de la herramienta Secaba-Rank para un mapa de la lectura pública a nivel de comunidades autónomas (2015)

\begin{tabular}{|c|c|c|c|c|c|}
\hline Red & Pres pc & Circ pc & $\mathbf{P}($ sis $)$ & Ranking & Estado \\
\hline Comunidad Foral de Navarra & 6.82 & 1.45 & 1.46 & 1 & \multirow{7}{*}{ Potencia } \\
\hline Región de Murcia & 4.31 & 0.86 & 1.37 & 2 & \\
\hline La Rioja & 7.22 & 1.42 & 1.36 & 3 & \\
\hline Castilla y León & 9.78 & 1.93 & 1.36 & 3 & \\
\hline Cataluña & 10.77 & 2.06 & 1.32 & 4 & \\
\hline Aragón & 7.62 & 1.29 & 1.17 & 5 & \\
\hline Principado de Asturias & 7.36 & 1.23 & 1.15 & 6 & \\
\hline Total Redes & 7.69 & 1.11 & 1 & 0 & Base \\
\hline Castilla-La Mancha & 10.11 & 1.4 & 0.96 & 7 & \multirow{10}{*}{ Dispotencia } \\
\hline Comunidad Valenciana & 5.83 & 0.8 & 0.95 & 8 & \\
\hline Cantabria & 6.1 & 0.83 & 0.94 & 9 & \\
\hline Illes Balears & 5.08 & 0.69 & 0.94 & 9 & \\
\hline Extremadura & 7.82 & 1 & 0.88 & 10 & \\
\hline Comunidad de Madrid & 8.54 & 1.08 & 0.87 & 11 & \\
\hline Galicia & 6.8 & 0.84 & 0.86 & 12 & \\
\hline País Vasco & 13.04 & 1.45 & 0.77 & 13 & \\
\hline Andalucía & 5.01 & 0.42 & 0.58 & 14 & \\
\hline Canarias & 4.61 & 0.34 & 0.5 & 15 & \\
\hline
\end{tabular}

Nota: Pres pc equivale a presupuesto per cápita; Circ pc a circulación per cápita; Form(sis) a la formulación del sistema; $\mathrm{P}($ sis $)$ a la potencia del sistema.

La metodología de la herramienta fue expuesta y puede ser consultada en Lázaro-Rodríguez et al. (2018), además de estar disponible en la sección de la metodología de la herramienta ${ }^{6}$. A su vez y como ya se dijo en la introducción, la metodología fue sometida a examen para probar su validez frente a otras técnicas como la del Análisis Envolvente de Datos (DEA) y el método Finlandia (Lázaro-Rodríguez, López-Gijón y Herrera-Viedma, 2019).

4. Acceso desde: http://secaba.ugr.es/rank/publicas/

5. Acceso desde: http://secaba.ugr.es/rank/publicas/index.php/estimacion-y-prediccion/

6. Acceso desde: http://secaba.ugr.es/rank/index.php/metodologia 
Aun con ello, cabe resaltar que la Potencia del Sistema es un indicador de segundo nivel que pone en relación las entradas de los sistemas (presupuesto), con las salidas (en este caso los préstamos). Así, se mide la eficiencia de las bibliotecas. A su vez, el ranking generado está basado en la Potencia de los Sistemas.

Por otro lado, el mapa de la lectura pública que se ofrece en la sección de Estimación y Predicción de la herramienta Secaba-Rank para las comunidades autónomas se basa en la relación entre el presupuesto y los préstamos. Así, la primera representación gráfica que se incluye en este trabajo consiste en el gráfico de dispersión entre las dos variables, esto es, en la representación gráfico del coeficiente de relación lineal entre las mismas. A ese respecto, cabe decir que en la sección de Estimación y Predicción de la herramienta Secaba-Rank Públicas se especifica que el resultado del coeficiente de correlación lineal de Pearson o Spearman según la normalidad o no normalidad de las variables respectivamente. Las variables de las comunidades autónomas en 2015 seguían una distribución normal, por lo que se utilizó el coeficiente de correlación lineal de Pearson. El resultado de dicho coeficiente fue de 0.78 y con significación estadística (con p-valor menor que 0.001). Por lo que la relación es significativa y positiva, lo que significa, sin ser una relación de causa, que sucede que a mayor presupuesto, mayores son los préstamos.

\section{Resultados:}

La primera representación gráfica es la basada en un diagrama de dispersión incluyendo una línea de tendencia (Figura 1). Dado que su naturaleza es dinámica, se recomienda acceder al enlace para su visualización en formato HTML. ${ }^{7}$

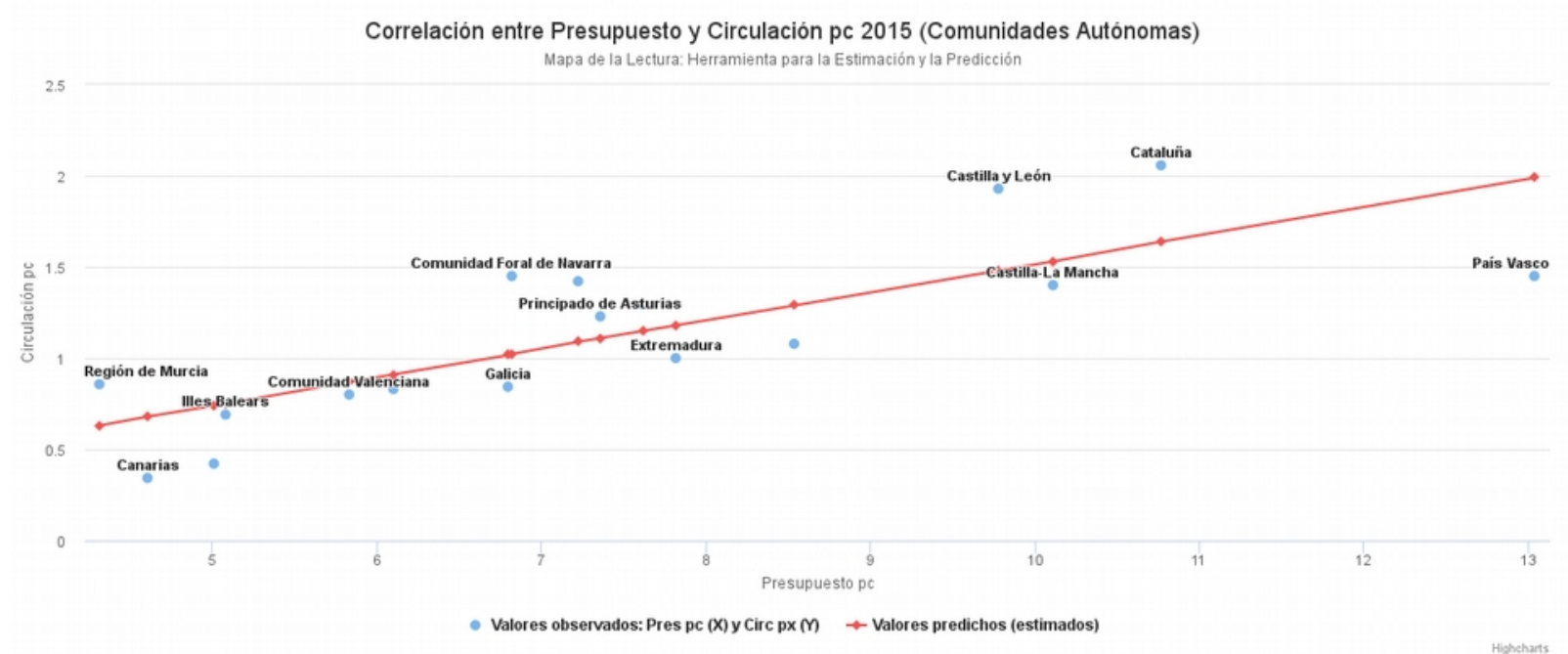

Figura 1: Mapa de la lectura pública 2015 a nivel de comunidades autónomas en Secaba-Rank Públicas. Fuente: ver enlace en nota 7 a pie de página

Como se puede ver, el eje horizontal representa el presupuesto per cápita, y el vertical, la circulación per cápita (préstamos). Se puede utilizar un zoom pinchando con el ratón o selector marcando la zona que se desea ampliar. Además, siempre se puede resetear el zoom para volver al gráfico en su estado original con la opción "Resetear zoom". Por último, en la leyenda se pueden

7. Acceso desde: http://secaba.ugr.es/rank/publicas/index.php/grafico-de-correlacion-entre-presupuesto-y-circulacionpc-2015-ccaa/ 
seleccionar los valores observados y los predichos (línea de tendencia) para verlos o no en el gráfico. De esta forma se pueden ver por separado.

La línea de tendencia en rojo marca el resultado para los préstamos esperados según cada presupuesto de cada sistema. Así, los sistemas que quedan por encima de la línea de tendencia, serían los que obtienen unos resultados mejores de lo esperado o estimado. Por su lado, los que quedan por debajo, serían los que obtienen un resultado por debajo de lo esperado. Los primeros son: Región de Murcia, Comunidad Foral de Navarra, Principado de Asturias, La Rioja, Aragón, Castilla y León y Cataluña. Son precisamente los que en la Tabla 1 obtenían una Potencia del Sistema mayor que 1 y un estado de potencia. Esto es, son los sistemas eficientes. De igual forma sucede con los sistemas dispotentes, que son: Castilla-La Mancha, Comunidad Valenciana, Cantabria, Illes Balears, Extremadura, Comunidad de Madrid, Galicia, País Vasco, Andalucía y Canarias.

El hecho anterior significa que la representación gráfica del mapa de la lectura pública según el diagrama de dispersión es una forma de representar al indicador de la Potencia del sistema. Los sistemas que más lejos quedan de la línea de tendencia por encima serían los más eficientes. Esto se ve muy bien en el gráfico en el caso de la Comunidad Foral de Navarra. Y lo mismo en el sentido negativo y de no eficiencia con los sistemas que quedan por debajo de la línea.

Por otro lado, la segunda representación gráfica se basa en un gráfico en movimiento a modo de circunferencia (Figura 2). Esta representación también tiene una naturaleza dinámica recomendándose acceder al enlace para verla en movimiento. ${ }^{8}$

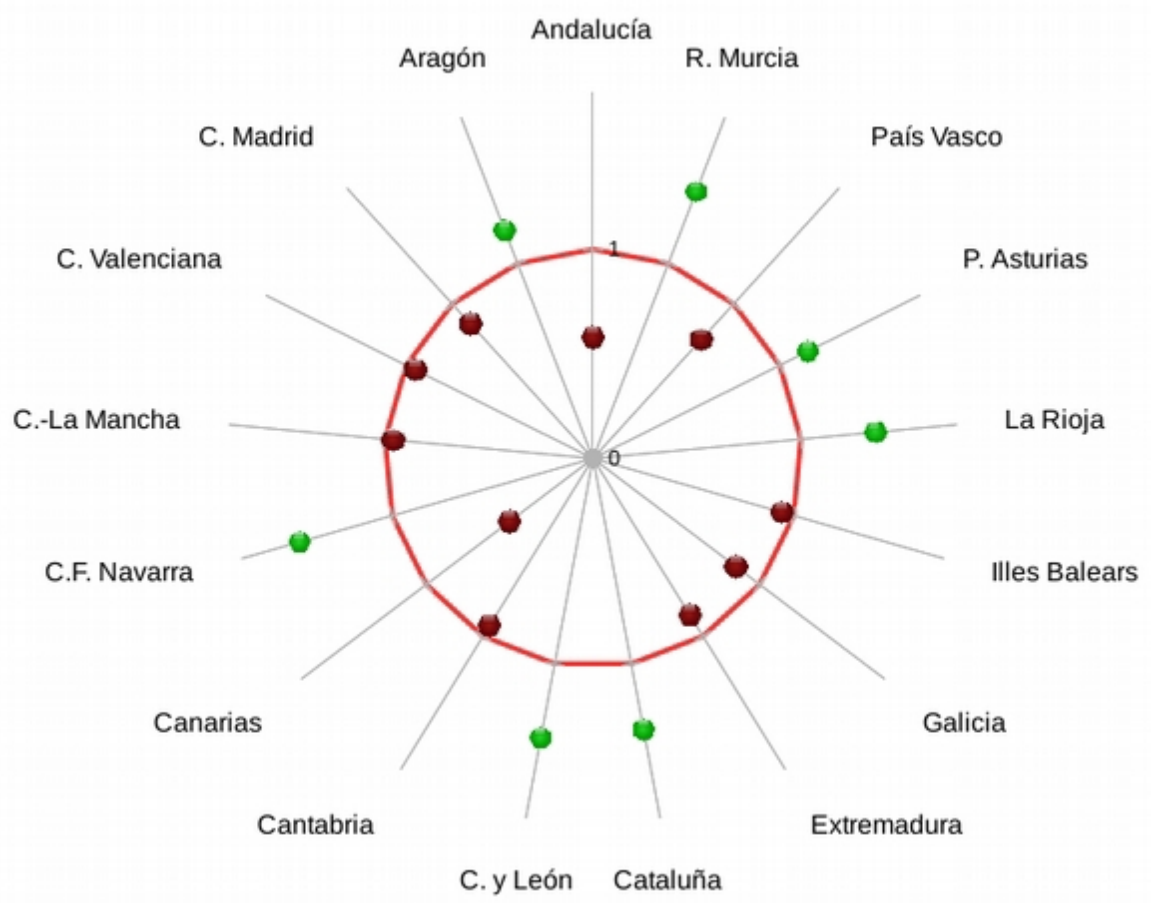

Figura 2: Captura de la representación gráfica en movimiento según el sentido de la circunferencia. Fuente: elaboración propia

8. Acceso desde: https://osf.io/vripw/ y https://osf.io/kmnct/ 
En este caso, el gráfico ha sido elaborado con el software de animación 2D para Linux de nombre Tupí 2D Magic Versión 0.2. ${ }^{9}$ Se toman en consideración los resultados del indicador de la Potencia del sistema. Así, la línea roja marcaría el resultado de 1, donde los sistemas tendrían un estado de sin potencia y punto a partir del cual un sistema sería potente (eficiente). Con todo, los sistemas que quedan por encima de la línea roja son los potentes, dibujándose con un punto en verde. Por su lado, los que quedan dentro de la línea roja, son los dispotentes o no eficientes, dibujándose con un punto rojo. El gráfico en movimiento simboliza el sentido de las agujas del reloj para los sistemas eficientes, y el sentido contrario para los no eficientes.

De la representación gráfica en movimiento cabe destacar que los sistemas con una Potencia del sistema mayor, recorren un mayor espacio en la misma unidad de tiempo que los sistemas con una eficiencia menor. A su vez, los sistemas no eficientes tendrían una eficiencia negativa según el sentido en que se mueven. Partiendo de la base de que todos los sistemas se considerarían en una misma unidad de tiempo, el espacio recorrido por cada uno podría calcularse y representarse según la fórmula siguiente:

$L=2 * \pi * r$

Donde:

$r=$ radio

$L=$ longitud

Así, si se considerase la Potencia del sistema como el radio, el sistema Comunidad Foral de Navarra, por ejemplo, con un radio de 1.46 obtendría una longitud o distancia recorrida aproximada de 9.17; el sistema Región de Murcia, de 8.60; el sistema Andalucía de 3.64, etc. De esta forma, se estarían expresando los resultados de la eficiencia matemática desde un lenguaje más matemático por medio de la fórmula de la longitud de la circunferencia.

La representación gráfica según la idea de circunferencia es justo el punto que se puede relacionar con la cuarta ley de Ranganathan sobre que las bibliotecas son un organismo en crecimiento (Ranganathan, 1931). Desde ahí, puede atribuirse la idea de expansión a los sistemas eficientes, pues su nivel de salidas es mayor que el de entradas; y la de implosión a los sistemas no eficientes, pues su nivel de salidas es menor que el entradas. Por último, una cuestión surge de todo ello: si algo a lo que nombramos como biblioteca, no cumple la ley de Ranganathan mencionada sobre que es un organismo en crecimiento, ¿podemos decir realmente que es una biblioteca? Una respuesta negativa conllevaría la consecuencia de que los sistemas de bibliotecas no eficientes podrían dejar de ser considerados como sistemas de bibliotecas.

\section{Discusión y conclusiones}

Este trabajo se basó en dar respuesta a tres preguntas de investigación. La primera y la segunda consistían en dar a conocer los resultados del análisis del mapa de la lectura en España ofrecido en la herramienta Secaba-Rank y en identificar qué redes, sistemas o bibliotecas resultaban por encima de lo esperado y cuáles por debajo en la relación entre entradas (presupuesto) y salidas (préstamos, visitas, etc.). Por su parte, la tercera planteaba cuáles pueden ser las ventajas de las nuevas formas de visualización de la información propuestas en el campo de las bibliotecas en particular y de la documentación en general.

9. Acceso desde: https://www.maefloresta.com/ 
En cuanto a la primera pregunta, los resultados se mostraron en la Tabla 1 añadiendo el presupuesto y la circulación per cápita, la Potencia de los sistemas junto con el ránking y el estado de los sistemas o redes. El análisis de los resultados se planteó desde la perspectiva de la visualización de la información y las nuevas formas de su representación para la mejor apreciación y entendimiento de la información. En cuanto a la segunda pregunta y a través de la primera representación basada en el gráfico de dispersión entre el presupuesto y la circulación, se vio cómo precisamente los sistemas potentes o eficientes según el indicador de la Potencia del sistema fueron los que resultaban en una situación mejor o por encima de lo esperado. Justo en el sentido contrario, se vio cómo los sistemas dispotentes o no eficiente fueron los que resultaron por debajo de lo esperado.

Por otro lado, en cuanto a la tercera pregunta de investigación, la elaboración de este trabajo es precisamente una apuesta hacia la mejor y más sencilla apreciación de los resultados sobre eficiencia que propone la herramienta y metodología Secaba-Rank mediante nuevas formas de visualización de información. Pasar de la representación estática de unos resultados en forma de tabla a su representación dinámica mediante los gráficos y las formas propuestas en este trabajo, se entiende como un paso adelante en la transmisión de resultados científicos y el avance de una disciplina o tema de estudio como la evaluación de bibliotecas.

En ese sentido, cabe resaltar la original propuesta que supone la presentación de la segunda forma gráfica de este trabajo. El gráfico en movimiento permite enlazar la no eficiencia de los sistemas dispotentes según la metodología Secaba-Rank con una de las leyes establecidas por Ranganathan para las bibliotecas, a saber, la de que son un sistema en crecimiento.

Por último, el hecho de que este trabajo se presentó como comunicación en las III Jornadas de Estudiantes de Ciencias de la Documentación, sirvió para poner a prueba la recepción de las representaciones gráficas propuestas. En ese sentido, cabe destacar que respecto de la primera representación gráfica se obtuvieron comentarios positivos, mientras que de la segunda hubo ciertas dudas sobre su sentido. Estas dudas servirán para replantear en el futuro la segunda representación gráfica del mapa de la lectura pública propuesto en este trabajo y para fortalecer la primera.

\section{Referencias}

- Chen, C. (2006). Information Visualization: Beyond The Horizon. Berlín, Germany: Springer Science+Business Media.

- Galán-Serrano, N., Lloret-Romero, N., Peset, F., y Torres-Faus, F. (2002). El mapa de lectura pública valenciana: un instrumento de trabajo y planificación. En I Congreso Nacional de Bibliotecas Públicas: La biblioteca pública: portal de la sociedad de la información (pp. 381-391). Madrid: Ministerio de Cultura, Subdirección General de Información y Publicación. Recuperado de http://hdl.handle.net/10421/1195.

- González-Rodríguez, C., Losa-Carballido, M-E, Fernández-Gutiérrez, B-E., VillanuevaFernández, C., Díaz-González, R-C., y Martínez-Marquínez, V. (2005). Mapa bibliotecario del concejo de Mieres. Aabadom: Boletín de la Asociación Asturiana de Bibliotecarios, Archiveros, Documentalistas y Museólogos, 16(1), 4-20. Recuperado de https://aabadom.files.wordpress.com/2009/12/2005-1mapabibliotecariomieres.pdf

- Lázaro-Rodríguez, P., López-Gijón, J., Alonso, S., Martínez-Sánchez, M.-Á., y HerreraViedma, E. (2018). Secaba-Rank, herramienta online para analizar y evaluar bibliotecas. El profesional de la información, 27(2), 278-288. doi: https://doi.org/10.3145/epi.2018.mar.06 
- Lázaro-Rodríguez, P., López-Gijón, J., y Herrera-Viedma, E. (2019). Secaba-Rank a examen: validación de su metodología sobre eficiencia en bibliotecas versus la técnica Data Envelopment Analysis (DEA) y el método Finlandia. El profesional de la información, v. 28, n. 3, e280316. doi: https://doi.org/10.3145/epi.2019.may.16

- López-Gijón, Javier (2003). La red de lectura pública de Andalucia: un análisis sistémico. (tesis doctoral). Universidad de Granada, Granada. Resuperado de http://digibug.ugr.es/bitstream/10481/29141/1/Lopez-GijonJavier.pdf

- Portús-i-Vinyet, M.D. (1999). El Mapa de la Lectura Pública de Catalunya: un instrument de planificació i d'avaluació. En Jornades Catalanes de Documentació, Barcelona, Collegi Oficial de Bibliotecaris $i$ Documentalistes de Catalunya. Recuperado de http://www.cobdc.org/jornades/7JCD/forum2.pdf

- Ranganathan, S. R. (1931). The five laws of library science. India: Madras Library Association; London: Edward Goldston.

- Yau, N. (2013). Data Points: Visualization That Means Something. Indianapolis: John Wiley and Sons. 